\title{
Der neue Schweizer Weltatlas
}

\section{Neuerungen}

Die Konferenz der kantonalen Erziehungsdirektoren hat 1979 einen gemeinsamen Atlas für alle Schulstufen und in drei Landessprachen beschlossen. Damit fand die Parallelität von Sekundar- und Mittelschulatlas ein Ende. Die finanziell günstigere Lösung fordert allerdings ihren Preis in Kompromissen bei der Auswahl der Karteninhalte, der Lesbarkeit und der geographischen Schwerpunkte. Dementsprechend sind nicht alle Karten für alle Stufen gleich gut geeignet. Es ist auch nicht so, daß der Atlas wie vom Nahen zum Fernen auch vom Einfachen zum Schwierigen aufgebaut ist. Vielmehr wurde darauf geachtet, in allen Teilen der Erde verschieden anspruchsvolle Karteninhalte präsentieren zu können. Um diesen Ansprüchen gerecht zu werden, wurde der Umfang des Atlaswerks stark erweitert: Die Anzahl Kartenseiten stieg (gegenüber der Ausgabe 1981) um 20\% auf 176, die Kartenzahl um $30 \%$ auf 362 , die totale Kartenfläche, dank breiterem Format, gar um 50\%.

Interessant ist in diesem Zusammenhang vielleicht auch die Kostenentwicklung: Kostete die erste Atlasausgabe 1915 noch 6 Franken, stieg der Preis bis zum Ende des Zweiten Weltkrieges auf 15 Franken (FREI 1948), während sich das schweizerische Nettoeinkommen parallel dazu verdreifachte. In den letzten fünfzig Jahren (von 1945 bis 1994) stieg der Schulpreis auf 45 Franken, die Inflation betrug im gleichen Zeitraum aber 413\%. Kurz: der Atlas wurde immer größer und für den Benutzer immer billiger.

Wer den neuen Atlas durchblättert, erkennt sofort, daß die Neuausgabe vielfarbiger, aber nicht etwa bunter erscheint. Die vielen topographischen Karten, welche in ihren Farbabstufungen ausschließlich das Relief darstellten, sind von sogenannten Übersichtskarten abgelöst worden. Hier spielt gegenüber der Oberflächenform die Oberflächenbedeckung eine entscheidendere Rolle. Wir sehen ein naturnahes Bild mit genaueren Hinweisen zur Vegetationszone. Diesen Karten ist im gleichen Maßstab (in Europa 1:4 Mio., in den anderen Erdteilen meist 1:15 Mio.) eine Wirtschaftskarte gegenübergestellt, welche funktionale Zusammenhänge aufzuzeigen vermag. Diese Teilung von Übersicht und Wirtschaft wurde, wenn immer möglich, auf einer Doppelseite realisiert (Beispiel: Griechenland oder Indien) oder bei größeren Ausschnitten unmittelbar angeschlossen (Italien oder China). Damit kann die Wirtschaftskarte stark entlastet werden, indem dort nur noch Orte bezeichnet sind, welche eine wirtschaftliche Bedeutung haben (u. U. zusätzliche für spezielle Lokalitäten wie etwa Industriestandorte), oder nur für den Güterverkehr wichtige Infrastrukturen eingetragen sind.

Neben dieser Hauptcharakteristik im neuen Schweizer Weltatlas ist die Ausweitung der thematischen Karten augenfällig. Dabei sind einfachere Detailkarten zu finden (z. B. S. 7-9 oder S. 33-40) wie auch anspruchsvolle (z. B. S. 25-27 oder S. 148-152). Die Themen sind stark physisch-geographisch geprägt: es gibt 63 Klimakarten (und darüber hinaus $82 \mathrm{Klimadiagramme)} \mathrm{sowie} 25 \mathrm{Karten}$ mit geologischer Thematik; daneben sind 40 Städtekarten und 26 «übrige» zu finden, mit primär (land)wirtschaftlichen Inhalten.

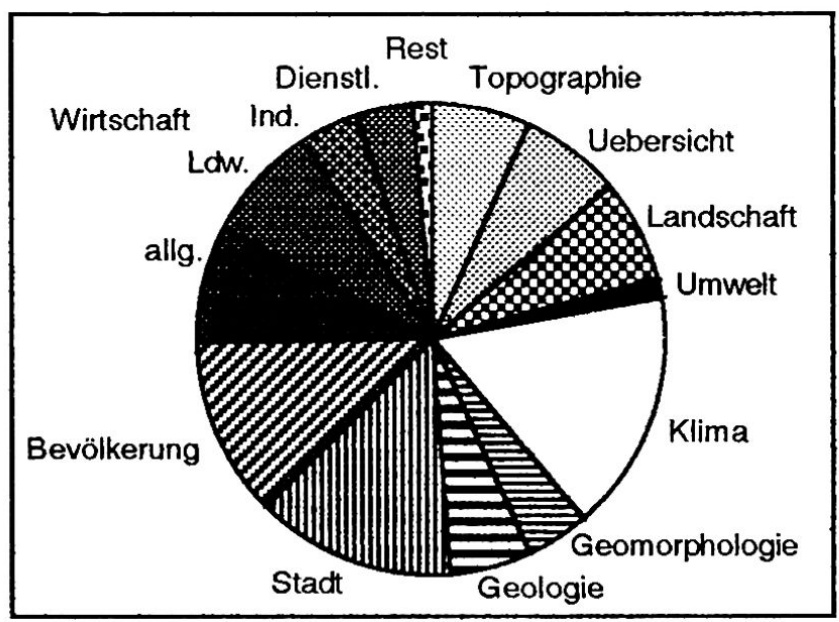

Abb.1 Kartenanteile.

Die Abb. 1 zeigt den Anteil der verschiedenen Themen in bezug auf die Kartenzahl. Ein Vergleich bezüglich der beanspruchten Kartenfläche würde eine deutliche Verschiebung zu den häufig doppelseitigen Übersichts- und Wirtschaftskarten ergeben und die meist sehr kleinen Klimakärtchen schwächer gewichten.

Roger Meier, Dr., Bahnhofstraße 40, 5400 Baden 


\section{Der Atlas als Lehrmittel}

Dem Schweizer Weltatlas kommt eine große Bedeutung bei der Schulkarriere zu. Er löst am Ende der Primarschule die Schulkarte ab, dehnt das Kartenlesen auf den Seiten 2 und 3 auf alle Maßstäbe aus. Die Besonderheit der Karte als Vertikalprojektion wird durch eine Gegenüberstellung einer Schrägaufnahme dargelegt. Im folgenden wird der primär topographische Karteninhalt durch immer mehr zusätzliche Angaben ergänzt (Denkmäler, landwirtschaftliche Sachverhalte). Der Atlas führt nach und nach in gleichbleibender Manier und Legende in die verschiedenen Regionen und Themen ein. Die vielen Detailkarten erlauben den Lehrkräften, individuell Akzente zu setzen, ohne einem übergeordneten, gesamtschweizerischen Lehrplan verpflichtet zu werden. Im allgemeinen werden die Karten mit höherer Seitenzahl komplexer, aber immer sind einfache Abbildungen eingestreut, wie etwa Naher Osten (S. 98/99), der Panamakanal (S. 138) oder die Erdübersicht (S. 156/157).

Mit Hilfe von 1000 Stichwörtern lassen sich aus dem Sachwortregister korrespondierende Themen zusammentragen. Von den Schülern wird vor allem der Namenindex rege benützt, um die Lokalität mittels ihrer geographischen Länge und Breite im Atlas zu suchen.

\section{Arbeitsweise}

Die folgende Beschreibung soll Ansätze zum Umgang mit dem Atlas und mögliche Lernschritte zeigen. Je nach Schulstufe ist dafür recht viel Zeit einzusetzen, um die Sicherheit im Gebrauch des Buchs zu erlangen und die Kompetenz zu festigen.

Eine große Schwierigkeit liegt in der großen Informationsfülle, welche sehr präzis auf kleinem Platz zu finden beziehungsweise zu suchen ist. Die Legende muß dazu sehr genau gelesen werden. In vielen Fällen wäre es sinnvoll, einen Teil der Karte abzudecken, eine Art Fenster zu schaffen, um die Konzentration auf die zu betrachtende Region zu lenken (Abb. 2). Ein Versuch mit einem

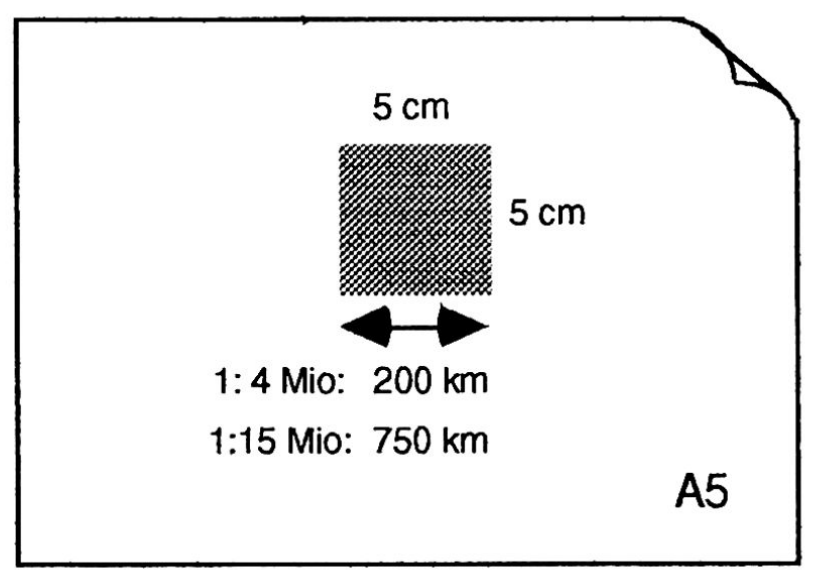

Abb. 2 Fenstermethode.
$5 \times 5 \mathrm{~cm}$ großen Fenster in einem A5-Loseblatt lohnt sich. Damit kann etwa die Vielfalt an Industrien und ihre Abgrenzung im Ruhrgebiet auf S. 50 deutlich gemacht werden, ohne daß man von der wirtschaftlichen Konzentration in Mitteleuropa abgelenkt wird. Dieses Fenster wäre auch geeignet, Galizien (S. 74) oder Guatemala (S. 143) isoliert zu betrachten und zu vergleichen, denn die Signaturen sind durch den ganzen Atlas dieselben und haben bei gleichen Maßstäben gleiches Gewicht. Diese Systematik sollte unbedingt genutzt werden für Vergleiche, etwa zwischen dichtbesiedelten Teilen Rußlands (S. 58) und Chinas (S. 119).

Die regionale Betrachtungsweise tritt gegenüber der thematischen und vergleichenden zurück. Selten geht es noch an, im Stoffprogramm länderkundlich vorzugehen. Die Geographie zeigt ja gerade in der Verknüpfung von Sachverhalten und im Vergleich von Regionen ihre Stärke. Wenn das Ruhrgebiet behandelt wird, ist es sinnvoll, dasselbe mit ähnlichen Industrieregionen in Europa zu vergleichen. Die Industriekapazität kann berechnet werden, die Tiere können sogar ausgezählt werden; nur gerade die Fische nicht!

Die Generallegende, die zu diesen Schülerarbeiten notwendig ist, kann aus der Seite 175 herausgeschnitten werden und läßt sich damit in jede Karte "einfügen». Sie erleichtert auch die Differenzierung der z. T. kleinen Farbnuancen in den Übersichtskarten, indem die Farbkästchen nahe an die fragliche Stelle herangeführt werden können (zu diesem Zweck wurden die Spalten in der nachgeführten Ausgabe 1994 vertauscht). Auch als Buchzeichen beim Arbeiten mit zwei Karten ist die herausnehmbare Legende nützlich.

In den Wirtschaftskarten bedeuten gleiche Signaturformen immer auch gleiche Rohstoffkategorien: stehende blaue Quadrate sind Erze bzw. übereck dargestellte Edelmetalle und Edelsteine, Barrenformen bedeuten übrige Bergbauprodukte, Raster und Bildsignaturen sind für Nutzpflanzen reserviert, Dreiecke gehören zum Thema Energie usw. Die Schülerin kann so z. B. gezielt aufGoldsuche gehen, indem sie die Karte nach gelben Quadraten absucht. Das Auge wird zunehmend trainiert für das gefragte Objekt, die Fülle an Information ist je länger, desto belangloser für die Fündigkeit. Auch in anderen Bereichen kann der Schüler die Einheitlichkeit der Signaturen nutzen (vgl. BRODERSEN 1986). Vertikal verflochtene Industrien sind mit einer gemeinsamen Farbe versehen. Damit ist die Standortsituation, etwa in der Metall-oder Ölindustrie, transparent zu machen. Die relevanten Zeichen werden beim Suchen mehrfach beachtet (vgl. SPIESS 1986). So baut die Schülerin die Zusammenhänge ständig aus, repetiert mit den Augen und wird den Karteninhalt so optimal speichern. Es ist nicht anzunehmen, daß in einfachen Karten, deren Inhalt sofort klar erkennbar wird, die Aussage längerfristig aufgenommen, erlebt, erlernt und memoriert wird. So erzeugen computergestützte Wirtschaftskarten (PC-Globe oder Mac-Globe) zwar nach langwierigem Aufstarten des Programms und Anklicken der gefragten Produktepalette eine übersichtliche Karte; aber es kann immer nur ein Produkt betrachtet 
werden, und die Darstellung ist dermaßen einfach, daß nicht viel Zeit auf das Studium des Inhalts verwendet wird. Die Kenntnisse gehen damit sehr schnell, meist schon mit dem Abschalten des Computers, wieder verloren. Häufig stehen die Schüler dem komplexen Karteninhalt des Schweizer Weltatlas am Anfang "einsichtslos» gegenüber, lernen aber um so nachhaltiger, mit dem Material umzugehen.

\section{Unterrichtsbeispiele}

Exemplarisch soll gezeigt werden, wie vielschichtig die Karten im Unterricht eingesetzt werden können. Dabei ist bewußt eine thematisch und regional breite Auswahl getroffen worden, die verschiedenen Inhalten und Schulstufen Rechnung tragen soll.

\section{Landwirtschaftsformen in Afrika}

Wir benutzen im Atlas die Seite 93, um am Beispiel Angola die Zusammenhänge zwischen Landschaftszonen und landwirtschaftlichen Möglichkeiten aufzuzeigen. Die Feuchtsavanne (vgl. S. 92) wird dem Wald zugerechnet und grün dargestellt, der Trockenwald der Trockensavanne erscheint in Oliv, die trockeneren Teile (Dornsavanne und Wüste) sind beige wiedergegeben. Nun ist aber nicht nur das Kulturland braun und mit Rastern und Signaturen der wichtigsten Produkte versehen, sondern auch die Landnutzungsform differenziert herausgehoben (Abb. 3). Der Schüler kann bestimmte Wirtschaftsformen mit landschaftlichen Gegebenheiten in Verbindung bringen. Die Küstentrockenheit (S.90) kann hinterfragt werden (Benguelastrom S. 158), die regenärmeren Landschaften gegen Süden und ins Landesinnere können mit vermehrter Viehwirtschaft korreliert werden. Das Beispiel soll demonstrieren, wie wichtig und wertvoll das Blättern und Nachschlagen im Atlas ist. Es darf nicht erwartet werden, daß alle Bezüge auf der einen oder

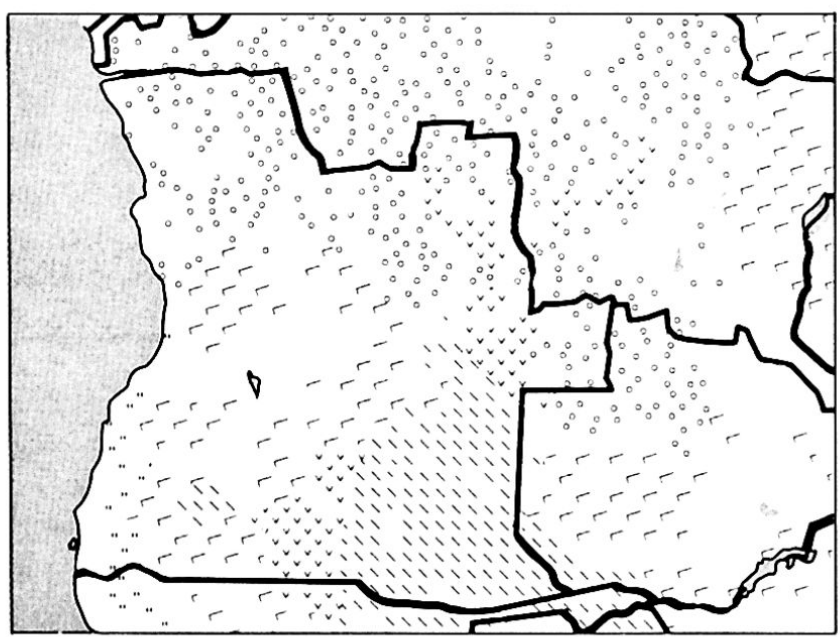

Abb. 3 Nutzungsformen in Angola (Auszug der Afrikakarte von S. 93, Schweizer Weltatlas 1993). andern Doppelseite geschaffen werden können. Sobald die Lektion auf einer Karte verhaftet ist, bleiben auch die Schülerinnen auf dieser Seite stehen, ohne die für die Geographie so wichtigen Querverbindungen zu suchen. Erst wenn sie selbst zu blättern beginnen, ist ein Lehrziel erreicht, nämlich die Neugier zu wecken und die Zusammenhänge selbständig zu entdecken. So kann bei einem Vergleich mit der Bevölkerungsdichte von S. 96 herausgearbeitet werden, wie der Hackbaubereich eine bessere Ernährungsbasis bildet als die Weide- und Jagdwirtschaft, denn er korreliert mit größeren Bevölkerungskonzentrationen (in Abb. 4 angepaßt an die Abb. 3 im Maßstab 1:30 Mio.).

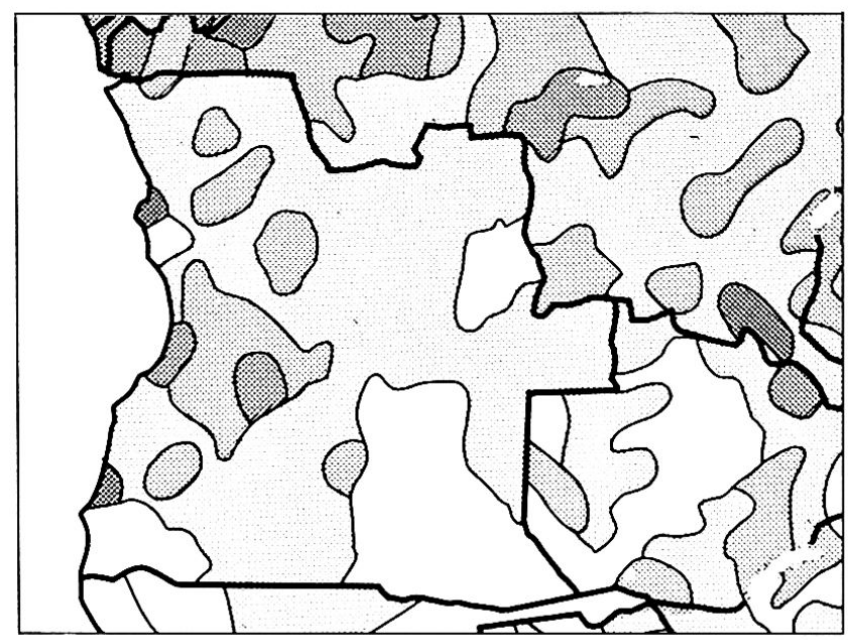

Abb. 4 Bevölkerungsdichte in Angola im Maßstab 1:30 Mio.; von 50-100 (dunkel) abgestuft zu 0-1 Einw. $/ \mathrm{km}^{2}$ (hell).

\section{Stadtentwicklung Paris}

Die kleinmaßstäbige Karte oben auf S. 33 zeigt schon im Überblick zwei Zeitschnitte zum Wachstum von Paris. In der unteren Karte kann der zentrale Bereich dann genauer studiert werden: Die Insel mit der Notre-Dame wird als Kern erkannt; hier, wo eine Überbrückung der Seine am einfachsten ist, liegen auch wichtige alte Gebäude der Stadt (Kathedrale, Palast, Rathaus, Universität). Die Boulevards Strasbourg und St-Michel bilden die Hauptachse von Norden nach Süden. Diese Verbindung ist schon in römischer Zeit angelegt worden. Die Karte des 12. Jahrhunderts (Abb. 5) zeigt ein Netz, das auch in der Atlaskarte noch Gültigkeit hat. Die mittelalterliche Stadt weist eine runde Form auf, die jahrringartig wächst, das Hôtel des Invalides und die Bastille bilden westliche und östliche Tangenten. Die Stadt wächst in der Neuzeit weiter, Arc de Triomphe und die Nation sind die neuen Außenmarken des Stadtrings; auf diesem verläuft wie auf dem inneren die tangentiale Metrolinie. Im 19. Jahrhundert wächst Paris bis zur heutigen Stadtgrenze, und durch Haußmann passiert eine starke Umgestaltung in dieser Stadt: verschiedene Avenues und Boulevards werden neu durch das Häusergewirr gezogen (BLUM 1994). 


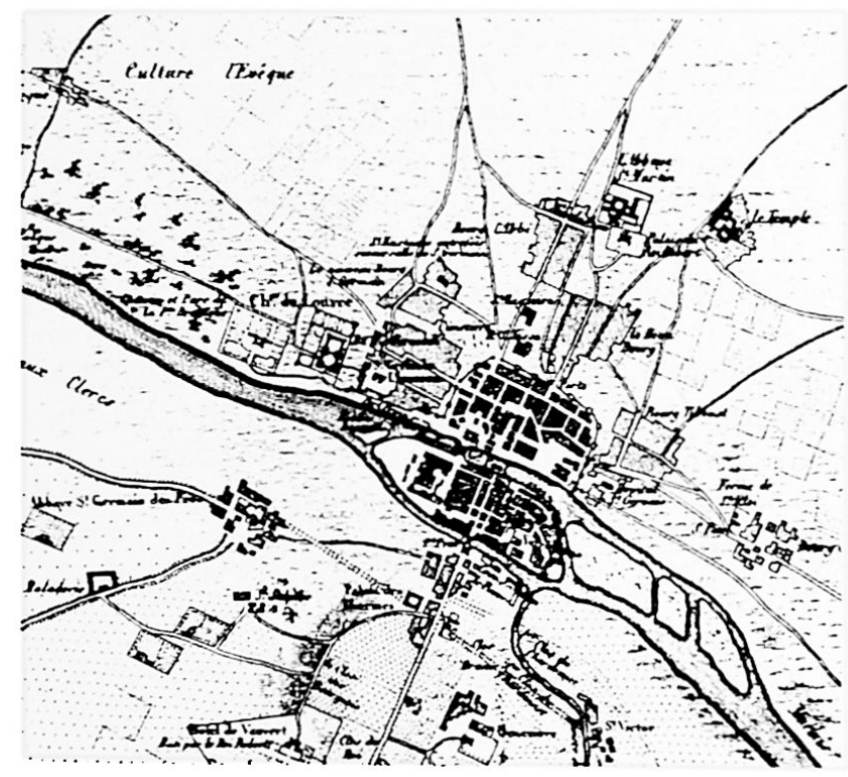

Abb. 5 Paris im 12. Jahrhundert (Aus: Process: Architecture No. 83).

Diese Straßenachsen und Sternplätze werden bald in allen Weltstädten modern. Solche neuen Straßen, wie etwa die Avenue de l'Opéra (vom Louvre zur Oper), sind daran zu erkennen, daß sie quer in der Wachstumsstruktur der Stadt liegen. Den Schülern müßte auch die Aufzählung einiger Sternplätze möglich sein. Auch ist die Lage der Bahnhöfe zu bestimmen und damit deren Alter.

\section{Die Verwendung der Klimakarten}

Eine Möglichkeit, die Einflüsse des Sonnenstandes auf die klimatischen Verhältnisse zu studieren, besteht darin, ein meridionales Profil anfertigen zu lassen (Abb. 6). Die Schüler lernen, mit Isothermen und Isohyeten umzugehen, und bemerken, daß diese im Zweifelsfall genau wie Höhenkurven abzuzählen sind: 25, 20, 15, 10, 15, 20. Die Abb. 6 ist auch gut dafür zu verwenden, die Kontinentalität und die Ursachen der Niederschläge beziehungsweise der Trockenheit zu erklären. Eine Umkehr der einen oder anderen Skalierung, d. h. die Spiegelung

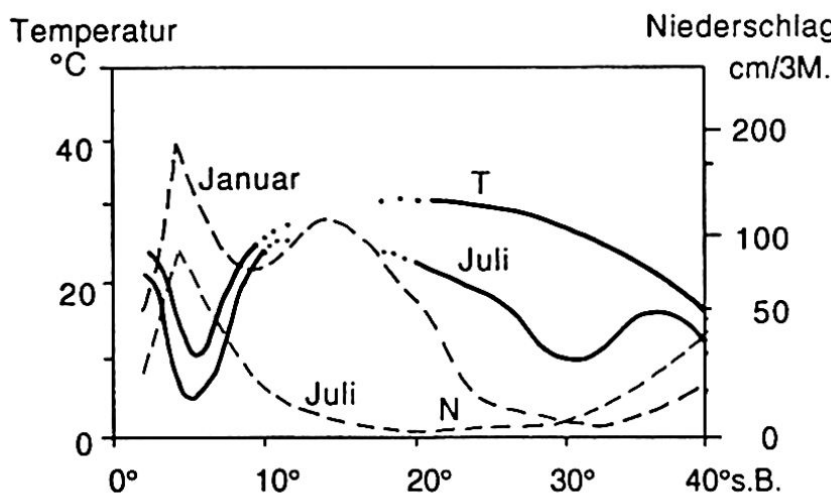

Abb. 6 Profil entlang dem 140. östlichen Längengrad. der Temperatur- oder Niederschlagskurve, könnte auch die Zusammenhänge zwischen diesen beiden Klimaelementen näherbringen.

\section{Plattentektonik}

Sollen die Schülerinnen mit der Karte S. 167 arbeiten, muß, wie schon bei den Wirtschaftskarten, von Anfang an klargemacht werden, daß hier vielschichtige Information steckt, die am besten durch gezielte Betrachtung einer Farbe oder Signatur reduziert wird. Hier könnte auch das Geoid studiert werden (Abb. 7), indem die Schüler nur die blauen Linien beachten, Maxima und Minima herausschreiben und sich mit den Zahlenunterlagen des Ellipsoides (S. 169) ein spezifisches Bild der Erde machen.

Die Information zur Plattentektonik ist vielfältiger. Relativ schnell erkennen die Schüler die Bewegungen und Geschwindigkeiten der Erdkruste, wesentlich mehr Mühe macht hingegen die unklare Abgrenzung der einzelnen Platten. Vielleicht könnten die ozeanischen Rücken und die Subduktionszonen in einer eigenen Farbe die Plattenränder deutlicher hervorheben. Die Flächenfarben der Karte zeigen die Sedimentierung während der Verschiebungen und beweisen damit Wegeners Theorie. Die Legende läßt sich mit Hilfe des Sachwortregisters mit Hinweisen auf verschiedene Detailkarten und der Darstellung auf S. 169 gut erarbeiten.

Damit ist natürlich nur eine kleine Auswahl aus den verschiedenen Themenbereichen in dieser didaktischen Vorstellung des Schweizer Atlas getroffen worden. Neben seiner Funktion als Nachschlagewerk und als Lehrmittel, wie hier hauptsächlich besprochen, kann er natürlich auch zur Prüfung oder Überprüfung dienen, etwa nach der Interpretation von Satellitenbildern. Die folgende Tabelle liefert vergleichbare Seiten des Schweizer Weltatlas und des Weltraumbildatlas (WESTERMANN 1981). Mit einem Klassensatz des Satellitenbildmaterials und Transparentpapier lassen sich so ausgezeichnet schülerzentrierte Arbeiten ausführen.

Viele Karten lassen natürlich auch viel didaktischen Spielraum offen. Der Kommentarband, der Ende Jahr zum Schweizer Weltatlas erscheinen soll, wird weitere Anleitungen und Anregungen geben.

\begin{tabular}{|l|l|c|c|}
\hline Region & Thema & WRB & SWA \\
\hline Wallis & Schneegrenze & 17 und 19 & 2 und 3 \\
\hline Lothringen & Versiegelung & 28 & 37 \\
\hline Rheintal & Vegetation & 30 & 38 \\
\hline Danziger Bucht & Kollektivierung & 45 & $52^{\star}$ \\
\hline Aetna & Lavaströme & 71 & 71 \\
\hline Athen & Stadtquartiere & 108 & $66^{\star}$ \\
\hline Nildelta & Besiedlung & 112 & $98^{\star}$ \\
\hline
\end{tabular}

Tab.1 Seitenvergleich zwischen Diercke Weltraumbildatlas und Schweizer Weltatlas. 


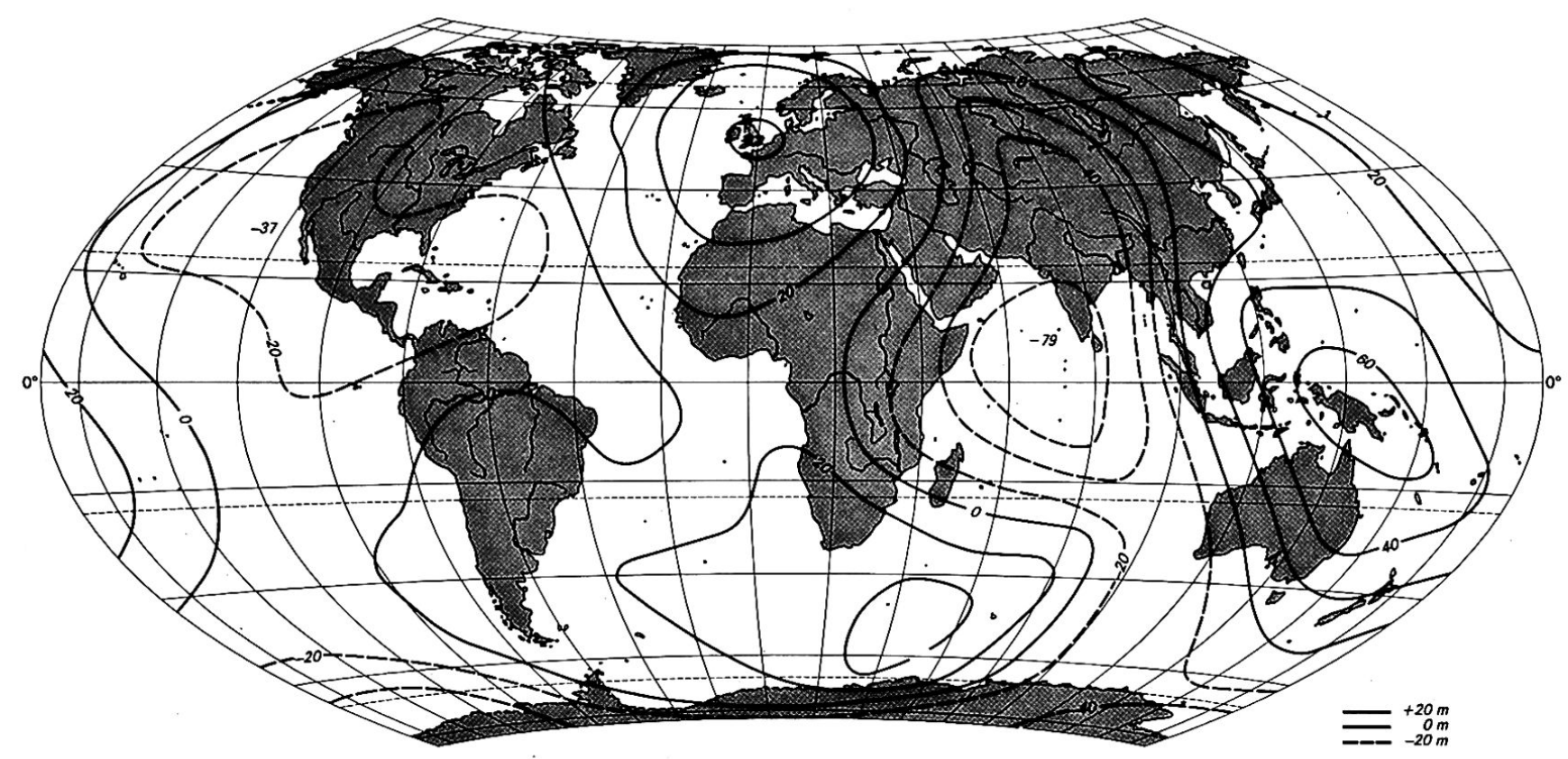

Abb. 7 Das Geoid (Auszug aus der Karte «Plattentektonik», S. 167, Schweizer Weltatlas 1993).

\section{Literatur}

BLUM, U. (1994): Städte in Vergangenheit und Gegenwart. In: "Geographie heute", Heft 120.

BRODERSEN, L. (1986): Aspekte der graphischen Gestaltung komplexer Wirtschaftskarten in Schulatlanten. Diss. ETH, Inst. f. Kartographie, Zürich.

FREI, G. (1948): Fünfzig Jahre Schweizerischer Mittelschulatlas. In: "Geographica Helvetica», III, Nr. 4.
SPIESS, E. (1993): Schweizer Weltatlas, Neuausgabe und (1994) nachgeführte Ausgabe.

SPIESS, E. (1986): Ansätze zur Interpretation thematischer Karten. In: Festschrift für G. Grosjean, Jahrbuch der Geogr. Gesellschaft von Bern, Band 55, Bern.

Weltraumbildatlas (1981): Diercke Westermann Verlag, Braunschweig. 